\title{
CONTENT STRUCTURE DESIGN OF NEW CENTURY EDITION OF THE NATIONAL FUNDAMENTAL GEOGRAPHIC ATLAS OF CHINA
}

\author{
Guizhi WANG ${ }^{1, *}$, Donghua WANG ${ }^{1}$, Junjun $\mathrm{QIAO}^{2}$, Wen $\mathrm{ZHOU}^{3}$, Mian CHEN ${ }^{4}$ \\ ${ }^{1}$ National Geomatics Center of China, 100830 Beijing, China - (wmj, donghua)@ngcc.cn \\ ${ }^{2}$ School of Geodesy and Geomatics, Wuhan University, 430079 Wuhan, China - jjqiao@ sgg.whu.edu.cn \\ ${ }^{3}$ Heilongjiang Administration Surveying, Mapping and Geoinformation, 150086 Haerbin, China - zhouwenaaa@163.com \\ ${ }^{4}$ Chinese Academy of Surveying and Mapping, 100830 Beijing, China - chenmian@ casm.ac.cn
}

Commission IV, ICWG IV/III

KEY WORDS: New Century Edition; The National Fundamental Geographic Atlas of China; The Theory of Compilation and Research; Content Planning; The Principle of Topic Selection; Structure Design; Map Arrangement

\begin{abstract}
:
The research and compilation of new century version of the National Huge Atlas of the People's Republic of China is the special basic work project by Ministry of Science and Technology of the People's Republic of China from June 2013 to May 2018. The National Fundamental Geographic Atlas is one of the important achievements, which represents the spatial distribution of basic geographic elements such as water system, landform, residential area, transportation, boundary, soil and vegetation in detail by relative balance. In order to meet the requirements of the construction of ecological civilization and the development of new urbanization, the set of surface coverage maps and the set of urban maps are added, and the new structure model of the National Fundamental Geographic Atlas is constructed. The Atlas is in 4-forma size, it is made up of five parts: group of sequence maps, group of topography and terrain maps, group of surface coverage maps, group of city maps and index of place names. This paper mainly discusses and studies the theory of compilation and research and content planning, the principle of topic selection and taking account of factors, structure design and map arrangement of Atlas.
\end{abstract}

\section{INTRODUCTION}

The research and compilation of new century version of the National Huge Atlas of the People's Republic of China is the special basic work project by Ministry of Science and Technology of the People's Republic of China. The project was officially launched in June 2013, under the auspices of the Ministry of Science and Technology of China. This project has carried out research on the overall design, database construction, product model and demonstration engineering, of the National Huge Atlas. Among them, The compilation and research of the National Fundamental Geographic Atlas is an important achievement of its research and construction.

The National Fundamental Geographic Atlas is an Atlas at the national level with provincial administrative divisions as the basic cartographic unit and fundamental geographical elements as the main content. It expresses in detail the spatial distribution of fundamental geographical elements such as water system, landform, residential area, transportation, boundary, soil and vegetation with relative balance. In order to meet the requirements of the construction of ecological civilization and the development of new urbanization, the set of surface coverage maps and the set of urban maps are added, and the new structure model of the National Fundamental Geographic Atlas is constructed.

It is the basic data for the national master plan, the development and utilization of land resources, the research of geosciences. It is also the important material for summing up the production and scientific research achievements of surveying and mapping and geography, as well as the education of patriotism.
As the first volume of the National Huge Atlas series, the National Fundamental Geographic Atlas is also the cartographic basis for compiling other atlases. According to the nature, task and object of the National Fundamental Geographic Atlas, this paper deeply carried out systematic research and structural design on the theory of compilation and research, the content planning, the principle of topic selection and taking account of factors, structure design, map arrangement and so on. The Atlas is in 4forma size, it is made up of five parts: group of sequence maps, group of topography and terrain maps, group of surface coverage maps, group of city maps and index of place names.

\section{RESEARCH THEORY AND CONTENT PLANNING}

This section mainly elaborates the theoretical basis, cultural concepts and design ideas of compilation and research of the Atlas.

\subsection{Theoretical Basis}

Atlas is a collection of maps with unified overall design, complete ideological system, systematic logical sequence, organic internal structure, and strict technical specifications. Its basic characteristic is systematicness, which requires people to have a strong systematic view in content planning, data analysis, theme selection, organization and arrangement of maps, etc. Materialistic Dialectics regards the objective world as a system of universal connection, which opens up a broad ideological realm for Atlas design and lays a philosophical foundation for the principle of atlas compilation. The system view mainly contains three meanings. 
2.1.1 Overall concept: Atlas is not a mechanical combination or simple patchwork of several maps, but a complete cartographic system with organic correlation and complementation. Any atlas has a clear orientation and theme, and its content planning must be based on this orientation and around this theme. The selected topics should be interrelated and assembled to form an organic whole according to a certain cartographic purpose and map use.

2.1.2 Comprehensive nature: Atlas should be able to comprehensively reflect the geographical environment, natural resources, social and economic phenomena of a region. In content planning, we should have comprehensive analysis ability of all directions and multiple perspectives, and take into account the factors of time series and spatial scale. Through comprehensive content combination, the relevant phenomena in cartographic areas can be comprehensively expressed.

2.1.3 Regional characteristics: Atlas is a systematic representation of regional environment, and regional characteristics are the unique scientific characteristics of atlas. In the design of atlas, firstly, the object represented by atlas, namely regional environment, should be studied in depth. Any region has distinct regional characteristics. when planning the content of atlas, we should fully consider these characteristics, so as to make the atlas more thematic and distinctive.

\subsection{Cultural Concepts}

From the point of view of the essential characteristics and practical value of maps, they all contain profound cultural connotations. Understanding and studying maps from the perspective of culturology has strategic significance for cartographic circles. It is very necessary to develop map ideas and give full play to the role of map in the field of human society and culture. Culture breeds maps. Maps are the product of culture Maps and culture are closely related. The topic selection, planning and structure design of atlas must have traces of cultural function and influence.

A good atlas design result depends not only on the designer's cartographic knowledge, but also on his cartographic cultural concept, cultural innovation consciousness, map cultural literacy and map cultural connotation, including rich map design experiments, as well as the understanding and application of natural environment characteristics, social and economic achievements, local knowledge, national customs and culture, the trend of development of the times, fashion aesthetic taste, etc.

The development of new technology enriches the manifestation of map, and also provides support for the innovation of map cultural products. This atlas adopts advanced technical means as far as possible, extensively acquires, fully considers and deeply excavates the cultural connotation of the cartographic area actively plans and conceives, carries out creative cultural design and cultural guidance, and effectively practices the role of editorial productivity and cultural productivity. The National Fundamental Geographic Atlas of China with a strong cultural concept can better represent the national level, have historical collection value, and better disseminate map cultural knowledge.

\subsection{Content Planning}

The compilation of this atlas is a complex system engineering, covering a wide range of contents, spanning large spatial scales and strong hierarchical structure. The natural and human geographical elements it expresses are a system of human-earth interaction, which needs to be managed by modern system engineering methods. The rapid development of modern science and technology, such as system theory, cybernetics and information theory, has fundamentally changed the process of human understanding and pushed it to a new level of development.

The content planning and structure design of this atlas always take the system view as the basic guiding ideology, fully realizing that the objective world is a universal integrated whole, in which all kinds of elements, things and phenomena are interrelated and interacted, and different regions have distinct regional characteristics. At the same time, based on the concept and innovative consciousness of modern map culture, makes a thorough study of China's basic national conditions and fashion aesthetic taste, and grasps the development trend of map culture in the new era.

This kind of design idea is mainly manifested in the top-down, left-right correlation, vertical and horizontal cross-cutting thinking process and mode of thinking, which runs through three levels of design.

2.3.1 Atlas as a whole: As the first level of planning, first of all, determine the nature, task and object of expression of atlas, grasp the accurate planning intention, and take general geographical elements as the main content. At the same time, for the integrity of atlas, there must be a comprehensive overview map and place name index. This atlas breaks through the traditional form. First, it strengthens the expression of provincial general geographical map, expresses basic geographical elements more clearly in the form of "topographic features + terrain elements" paired maps; second, it adds surface coverage maps and urban maps to highlight the theme of urbanization and ecological environment.

2.3.2 Each map group: As the second level of planning, give full play to the advantages of comprehensive analysis and expression of the series of maps in each map group, and reflect the differences and unity of mapping objects with a complete set of maps, which are comparable, complementary and clarified with each other. For example, the contents of each map in the sequence map group are different but all around the theme of basic geographical conditions; the series of maps in the topography and terrain map group represent different provinces, but all reflect the general geographical elements of each province.

2.3.3 Each map: As the third level of planning, each map is also a small system complex, with all elements connected to each other, reflecting the subject content of a map comprehensively. For example, the general geographical map takes the surface of the earth as a complete complex through its six elements, and the urban map takes each city as a small complex to systematically represent the relevant natural and human geographical information of the city.

\section{THE PRINCIPLE OF TOPIC SELECTION}

According to the nature, task and object of the National Fundamental Geographic Atlas, it is mainly a general map of basic geographic information represented by a smaller scale, including general map of the whole area, general map of large area, detailed zoning map, large-scale city map, etc.

In addition, there are a certain number of general thematic map as a sequence for people to understand the general geographical situation of the mapping area. The topic selection of this atlas mainly embodies the following characteristics. 


\subsection{Considering the Integrity of Environmental System}

Atlas is the systematic expression of a regional environment. When using atlas to express the internal structure and multi-level relationship of the environment, first of all, we should pay attention to the integrity and systematization of content selection. Integrity is not all inclusive. To show the integrity of a mapping area with a limited surface, it is necessary to make a scientific choice and choose, i.e. select the major and discard the minor.

This atlas focuses on the expression of basic geographic information content, which not only reflects the natural environment background, but also reflects the social and economic value created by the development, utilization and protection of the natural environment. From two aspects of multiscale general geographic map and general thematic map, it constructs the performance content of the atlas.

The general geographic map is the main content of the atlas, which represents the basic geographic information content such as water system, landform, residential area, traffic, boundary, soil quality and vegetation in detail and balanced as much as possible. The general thematic maps are the opening part of the atlas. Their main part are the national general maps of the general map elements, which comprehensively introduces China's geographical overview and construction achievements. Natural geography and humanity Geography is equally important, while avoiding duplication with other volume atlas contents.

\subsection{Pay Attention to the Logicality of Space-Time Relationship}

The map has the special expression ability of three-dimensional space. From the spatial scale, the atlas is divided into three levels: national, provincial and urban areas, gradually improving the resolution and refining the expression content. In the research and design of space-time relationship, the spatial scale and time series are fully grasped. Logicality is the systematic requirement of the topic selection and conception content in the Atlas planning.

The topic selection of the atlas is from high to low, from top to bottom. Without systematic analysis, there will be no time and space structure, and there will be no logical arrangement of maps. The logical thinking function of atlas can be enhanced by establishing the space-time relation system of atlas content with logical thinking.

There are many logical relations in the topic selection of this atlas, such as macro and micro, nature and human, important and secondary, comprehensive and special, current situation and planning.

This kind of spatiotemporal and logicality is not a simple correspondence, but a cross combination. Through analysing the topic connotation and mining the internal logic relationship, the organic combination of spatiotemporal and logicality can be realized.

\subsection{Reflect the times of social development}

This atlas tries to reflect the features of the times and the issues that people are eager to pay attention to. According to the development strategy and tasks of China's "one belt and one road", the marine economy, the integration of Beijing, Tianjin and Hebei, Guangdong, Hong Kong and Macau, the construction of ecological civilization, the monitoring of geographical conditions, and the acceleration of new urbanization, the corresponding theme contents were selected. Among them: the 39 selected central cities are the core of China's major economic regions and urban agglomerations, which can better play a supporting and leading role in optimizing the layout and form of urbanization, radiating the development of urban agglomerations, forming economic growth poles, etc.. Ecological environment topics include 10 kinds of land cover information, such as manmade surface, cultivated land, bare land, grassland, forest, shrub land, water body, wetland, glacier and permanent snow, tundra, etc. The determination of these topics adds color to the time sense of atlas.

\subsection{Give consideration to the knowledge of innovative design}

Innovative design is the source and power of Atlas Compilation and research. Content planning innovation is an arduous task. Because the topic content involves a large amount of multidisciplinary and cross industry information, it needs to refine, summarize and sublimate a large amount of words, pictures, statistics and other information.

Therefore, designers are required to have solid professional knowledge, give full play to the important role of knowledge, and have no limitations in the listing and perfection of things. We should pay attention to connotation analysis, highlight the theme, and directly point out the innovative ideas of problems.

This atlas uses innovative thinking to plan different topics, grasps the characteristics of the times and the development frontier, studies and researches China's current major development strategies and achievements, conducts research and in-depth study in relevant fields without departing from the general geographic theme, extracts the content indicators with profound meaning, sets a new school, highlights the key points, so as to more scientifically and effectively express the selected theme content, better approach the requirements of the times, and meet the needs of national economic development.

\section{STRUCTURE DESIGN AND MAP ARRANGEMENT}

Atlas is not a mechanical combination and simple patchwork of various maps, but a complete map system that is organically related and complementary to each other. It is a map aggregation formed according to certain cartographic purposes and purposes. Reasonable structure design can make the whole atlas have new functions different from each component in the process of operation.

The structure arrangement of any atlas must conform to certain internal logical relations, forming the logical order of induction and classification, deduction and analogy, cause and result, synthesis and decomposition, otherwise it will give people a sense of disorder.

Therefore, the atlas often shows a multi-level tree like space-time system structure. The design and arrangement of map group is an important part of the content framework design of atlas, not a simple listing and mechanical patchwork of content topics, but a systematic consideration and overall idea of the theme of atlas.

The most important characteristics of atlas are systematization and logicality, which are mainly reflected in the structural design of map groups and the logical arrangement of map sheets.

In structural design, the structure mode of "Atlas + group + sheet" is adopted. 
In map arrangement, it basically follows the arrangement principle of "first macro, then meso and micro; first natural, then human; first graphic, then text".

In consideration of the proportion, sequence and coordination of all kinds of maps, this atlas has always run through the systematic and logical ideas. It constructs the main frame structure of the atlas from two aspects of spatial zoom and content theme, which is obviously manifested in three administrative structures of whole country, province and city according to the spatial scale vertically, and classified in several categories according to the content theme horizontally.

The overall structure of the atlas is shown in Figure 1:

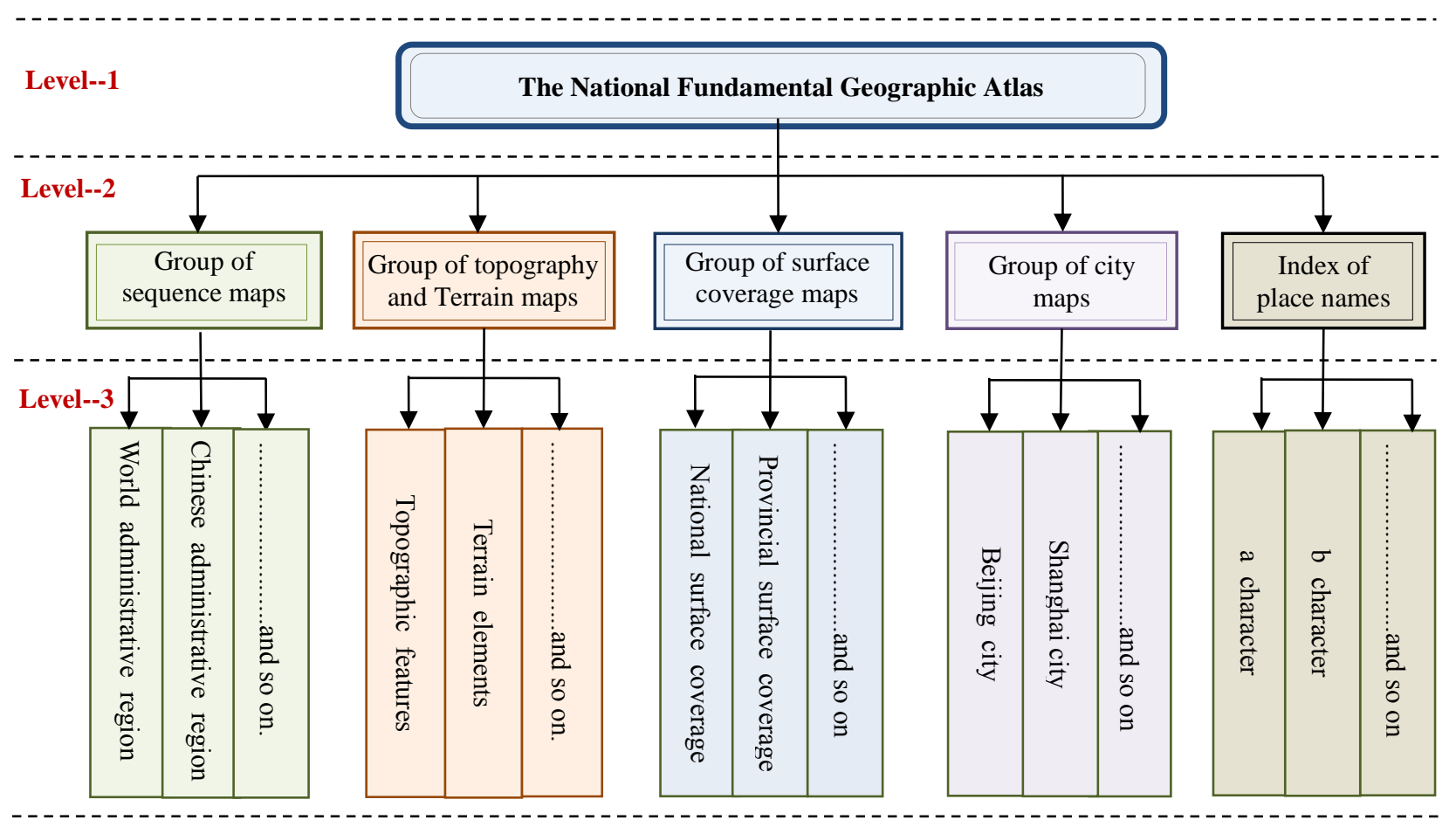

Figure 1. The general structure of the new century edition of the National Fundamental Geographic Atlas of China

\subsection{First Level Structure - Atlas}

The first level structure of an atlas is the atlas itself, which is composed of several map groups representing different themes. The content structure of each group and the arrangement and combination of them directly affect the overall quality and scientific value of the atlas.

This project constructs a new structure model of the National Fundamental Geographic Atlas based on spatial zoom and content selection. It has fully explored the advantages of spatial analysis and data combination of atlas database, and built a threelevel spatial content structure mode with basic geographical elements as the main body, provincial and regional maps as the emphasis, central cities as the reinforcement, and ecological environment as the emphasis.

This atlas is composed of 4 map groups and 1 index, including group of sequence maps, group of topography and terrain maps, group of surface coverage maps, group of city maps and index of place names.

They are separately the national general plan maps reflecting the overall situation, the provincial administrative area maps reflecting the basic geographical elements, the surface coverage maps reflecting the ecological environment, the city maps of provincial capital-level and specifically designated in the state plan reflecting the urbanization, and the index catalogue retrieving the geographical names above the township level.

In a mathematical language, the structure and function of atlas are expressed as follows: Therefore, the structural expression of an atlas can be written as:

$$
\mathrm{V}=\left\{A_{i}=\varnothing\left(T_{i}, N_{i}, H_{i}\right)\right\}, i \in n
$$

In the formula:

$\mathrm{V}$ is the atlas.

Ai is the group $\mathrm{i}$.

$\mathrm{Ti}$ is the time influence factor of the group $i$.

$\mathrm{Ni}$ is the natural factor of the group i.

$\mathrm{Hi}$ is the human factor of the group $\mathrm{i}$.

Each graph group of the National Fundamental Geographic Atlas starts with a representative title page. The sample structure of the atlas is shown in Figure 2: 


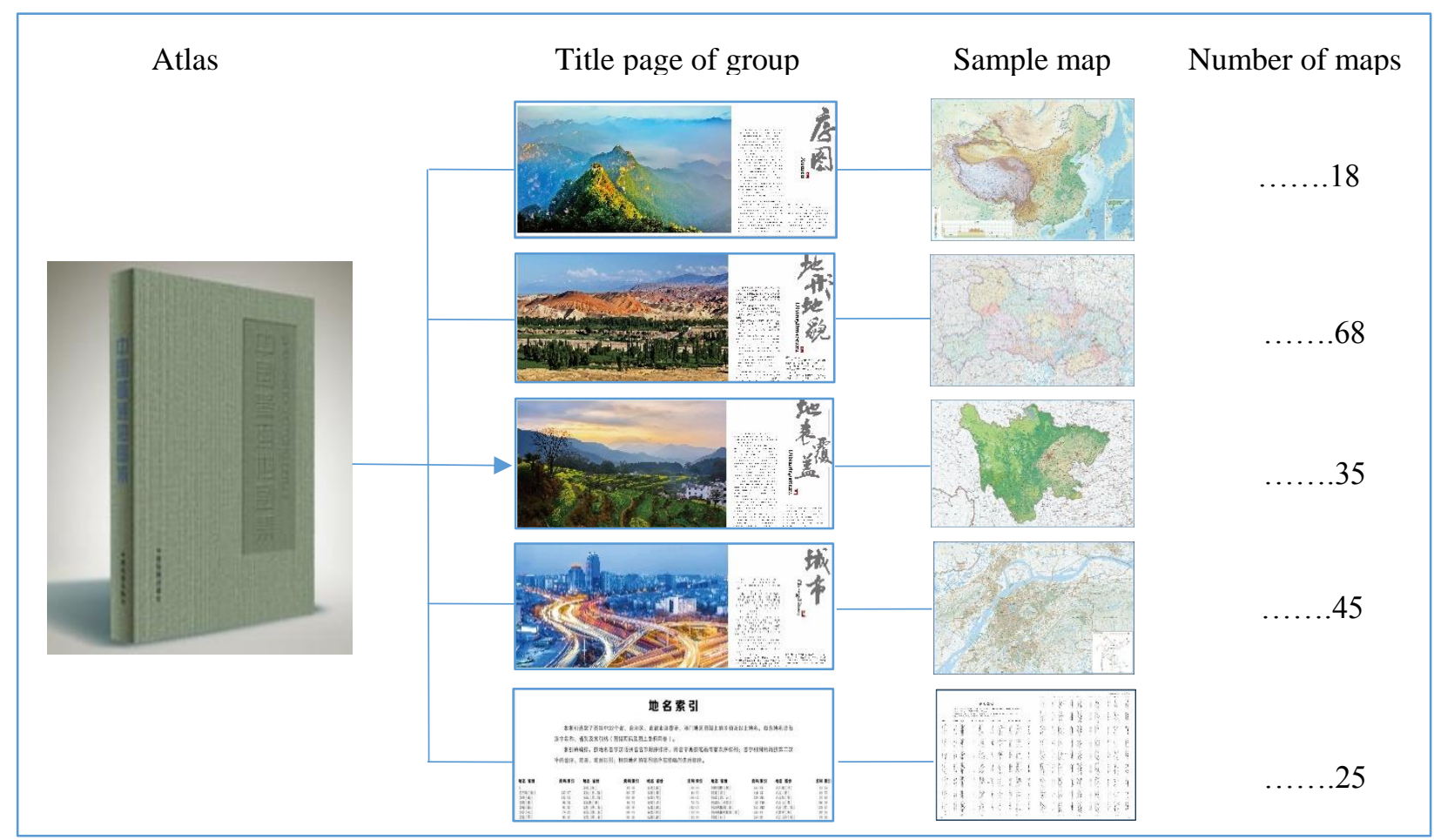

Figure 2. The sample structure of the National Fundamental Geographic Atlas of China

\subsection{Second level structure - groups}

In this atlas, the spatial scale is the main line, the thematic combination is the auxiliary line for logical arrangement, and the place name index is the last. On the spatial scale, with 3 visual field such as country, province and city, gradually improve the resolution, refine the representation content.

The spatial main line with clear effect structures is formed from the sequence map group of the world and China to the topography and terrain map group and the surface coverage map group of provincial administrative region, then the city map of provincial capital-level and specifically designated in the state plan.

In terms of thematic combination, the first is the sequence map group reflecting the overall situation, the second is the topography and terrain map group reflecting basic geographical elements, and the surface coverage map group reflecting ecological environment, the last is the city map group reflecting level of urbanization and urban construction, and the place name index retrieving the geographical names above the township level, formed a logical and orderly thematic arrangement auxiliary line.

Through such an orderly arrangement, we can build a large space-time system integrating geographical space, natural resources, economic construction and social development, fully grasp its time series and spatial scale, which is of great significance to reveal the laws of economic construction and social development. Therefore, the structure expression of a graph group can be written as:

$$
\mathrm{A}=\left\{M_{j}=\varnothing\left(P_{j}, N_{j}\right)\right\}, j \in n
$$

Where: $\mathrm{A}$ is a group, $\mathrm{Mj}$ is the map $\mathrm{j}, \mathrm{Pj}$ is the previous page influence factor of map $\mathrm{j}$, and $\mathrm{Nj}$ is the next page influence factor of map j.

The composition and functions of each group are as follows.
4.2.1 Group of sequence maps: As preface of the atlas, it has a strong macro and flexibility. First, it takes the world map as the prelude to give readers a complete global concept and the geographical location of our country. According to the spatial scale, we will arrange the "one belt and one road" area map. Then, enter the series of Chinese maps, first led by a vertical version of the whole picture of China, showing the whole picture of China integrating land and sea, followed by a total of 18 sequence maps of China's terrain, China's sea area, etc. In terms of content, it can be basically divided into guide map, natural geographic map, human geographical map, historical and cultural map and other types.

4.2.2 Group of topography and terrain maps: It is an important part of the Atlas, including 34 provincial cartographic units, which are 68 pictures in total. Each cartographic unit includes two basic combinations of "topographic features + terrain elements". The combination of two pictures more clearly represents the spatial distribution and interrelationof basic geographic elements. According to the order of the administrative division Manual of the people's Republic of China, starting from North China, opening with Beijing, it is followed by northeast, East China, Central South, southwest and northwest regions, and finally Hong Kong, Macao and Taiwan.

4.2.3 Group of surface coverage maps: Including two spatial scales of national and provincial administrative areas. In accordance with the order of the administrative division Manual of the people's Republic of China, with one national surface coverage map as the guide, Thirty-four provincial administrative area surface coverage maps are arranged in sequence. Each map is represented by a unified 10 categories of surface classification standards. 
4.2.4 Group of city maps: It is composed of 46 city maps of three spatial scales such as nationwide, key regions and important cities. From macro to medium and then to micro, 4 national city thematic maps, 3 key regions thematic maps, 39 important city maps are successively set up. The national city thematic maps are China's cities, famous cities and towns in China, China's urbanization, China's Urban Agglomerations, which show the spatial geographical location, urbanization development level, population scale, historical and cultural evolution of Chinese cities from macroscopic view. The key regions thematic maps are Beijing Tianjin Hebei, Yangtze River Delta and Guangdong Hong Kong Macao Bay Area, which reflect the all-round development and opening trend of key areas in China at medium level, from north to south. The important city maps are 34 provincial capital-level and 5 specifically designated in the state plan, which described the overall appearance and development of the cities in detail and are the main body content of city map group.

4.2.5 Index of place names: Place names are important geographic information and social public information, closely related to people's daily production and life, and play a wide role in national and social governance, economic development, cultural construction, national defense, diplomacy, etc. Place name index is a list of place names arranged in a certain order to retrieve the location of all kinds of place names on the map. It is a kind of file index which indicates place names and corresponding file numbers and is arranged in a certain order.

\subsection{Third level structure - Sheets}

Sheets are the most basic unit of the atlas, and there are often close connections between them. Each map of this atlas basically occupies an expanded page, and its content selection, drawing expression, editing and processing directly affect the accuracy and quality of the atlas. Therefore, the structure expression of a sheet can be written as:

$$
\mathrm{M}=\left\{E_{k}=\varnothing\left(I_{k}, P_{k}, L_{k}\right)\right\}, k \in n
$$

Where: $\mathrm{M}$ is the sheet, $\mathrm{Ek}$ is the element $\mathrm{k}, \mathrm{Ik}$ is the thematic information of the element $\mathrm{k}, \mathrm{Pk}$ is the graphic parameter of the element $\mathrm{k}, \mathrm{Lk}$ is the language and text parameter of the element $\mathrm{k}$.

According to the division of map groups, one or two typical sample sheets are selected to elaborate.

4.3.1 group of sequence maps: There are various kinds of diagrams, involving various indexes of common maps. Each diagram has a clear theme idea, only representing one theme content. Except for the public base map, the selection indexes, processing methods and editing principles of other theme content are different.

World map: This map has a novel appearance, a certain spherical sense and is close to the length width ratio of the rectangular frame. The area and angle deformation of the main continents in the world are moderate, and there is no obvious visual deformation of the main land shape. Moreover, China is in the middle of the map, the Pacific Ocean is complete, and South and North America graphics are not repeated. As shown in Figure 3:

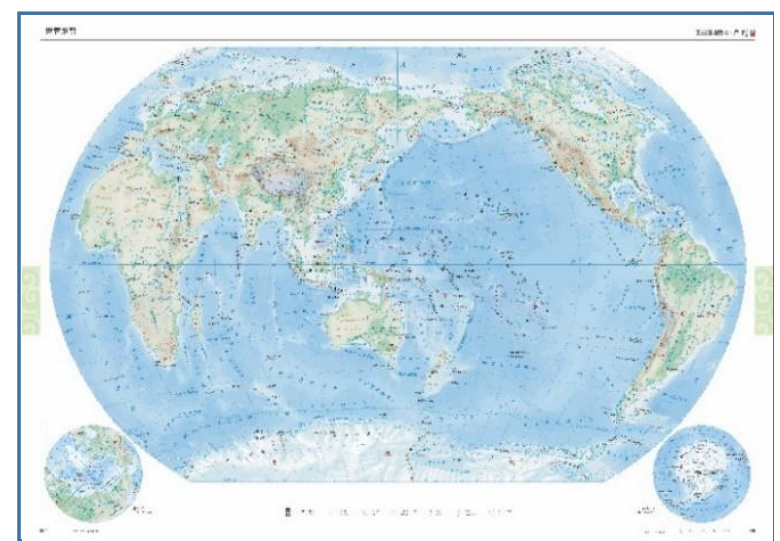

Figure 3. World Terrain Map in the Atlas

China map: Terrain map of China highlights the natural geographical elements such as topography, rivers and mountains, simply shows human geographical elements as auxiliary information, as shown in Figure 4.

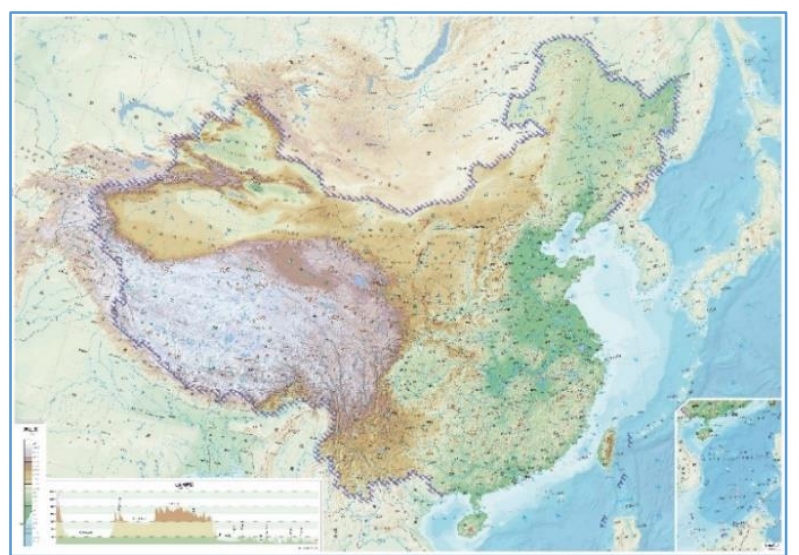

Figure 4. Terrain Map of China in the Atlas

4.3.2 group of topography and terrain maps: Each provincial administrative unit consists of 2 maps which are topographic features map and terrain elements map.

Topographic map: It focuses on the expression of human geographical features. It represents the administrative area and boundary, residential area, traffic, water system and other elements in detail, represents mountain, mountain, desert, green space and other elements in brief, and uses the gray shading as the background to express the terrain information. As shown in Figure 5:

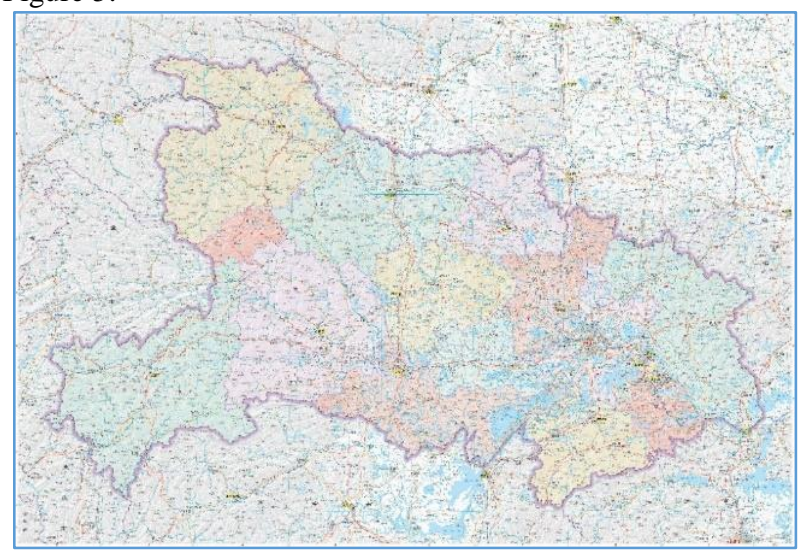

Figure 5. The topographic features map of Heilongjiang prov. 
Terrain map: It focuses on the expression of natural geographical elements such as topography, rivers and mountains, and scientifically expresses the content of terrain and geomorphology in the form of layering and coloring, while other humanistic geographical elements that play a role of positioning and reference are briefly expressed. As shown in Figure 6:

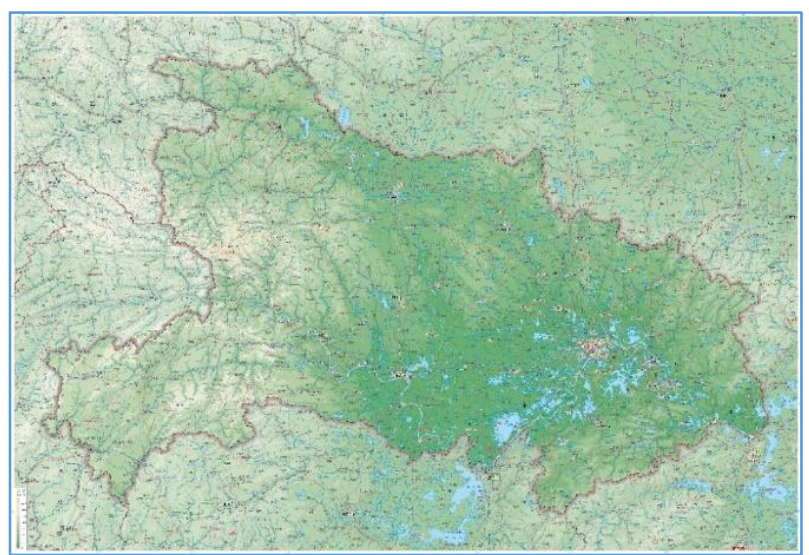

Figure 6. The terrain elements map of Heilongiiang province

4.3.3 group of surface coverage maps: According to the unified classification of surface cover, it uses the method of bottom material to represent 10 kinds of surface cover elements such as cultivated land, forest, grassland, shrubbery, wetland, water body, tundra, artificial surface, bare land, glacier and permanent snow, and roughly represents the basic geographical elements such as residential land, transportation, boundary and water system. As shown in Figure 7:

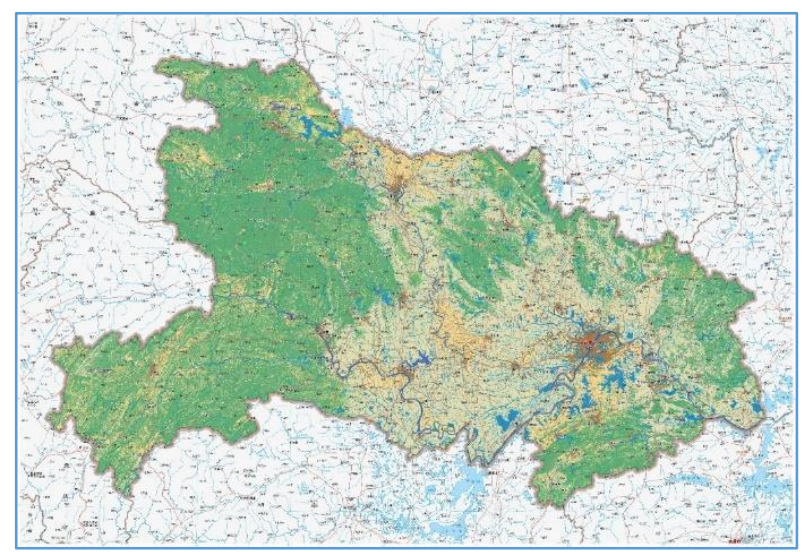

Figure 7. The surface coverage map of Heilongjiang province

4.3.4 group of city maps: The city map is an enlarged map of the topographic map. It should pay attention to the connection with the topographic map, mainly reflecting the plan outline, internal structure and external connection of the city. It express in detail the block, green space, water system, important public buildings, streets, railways, highways, government locations, important service facilities (including hospitals, hotels, banks, schools, petrol stations), important cultural relics and historic sites, tourist attractions, etc.

Due to the great changes in cities, it is necessary to update, supplement and refine the city map according to the highresolution image data, network map and tourism map.

The city maps improved the status of the traditional atlas which only represents the scope of the main urban area and is attached to the provincial map, creates the city map group, greatly expands the scope of the city map, completely expresses the overall appearance of the urban area, and constructs a complete and smooth new urban high-level road network based on the image and Internet, highlighting the spatial layout of the urban skeleton.

\section{As shown in Figure 8:}

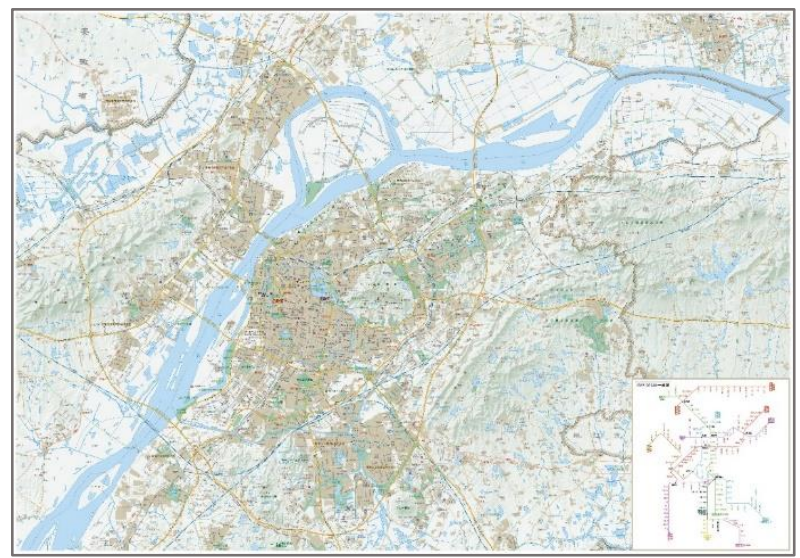

Figure 8 . The city map of Wuhan in Hubei province

4.3.5 index of place names: The national Toponymic index is arranged in the order of Chinese phonetic syllables of the first characters of toponymic Chinese characters, among which homonymic characters are arranged in the order of simple and complex strokes of Chinese characters. the same strokes of Chinese characters are arranged in the order of horizontal, vertical, apostrophe, point and fold strokes. If the first characters are the same, they are arranged according to the second Chinese character's phonetic order, stroke and pen order. If the first two Chinese characters are the same, they are arranged according to the third Chinese character's phonetic order, stroke and pen order, and so on.

\section{CONCLUSION AND PROSPECT}

Based on the theory of system and the principles of integrity, logicality, epochal and knowledge, the National Fundamental Geographic Atlas does well in the innovation planning of atlas, enhances the systematic concept of atlas, pays attention to the strategic height of atlas, emphasizes the resource advantage of atlas, and highlights the era characteristics of atlas. Through four thematic map groups and one place name index, this atlas comprehensively shows the basic geographic information and related thematic information which are interrelated and dense in content. The whole atlas has not only a clear vertical hierarchical structure, but also a wide horizontal logical sequence. The map groups are interconnected, and the map sheets are connected with each other, forming a complete integration of distinct levels, logical order, vertical and horizontal integration.

This project innovatively constructs a new structure pattern of large-scale general atlas based on high-performance information mining and layer data cluster expression of database, puts forward a design idea of "national general atlas" structure model with spatial zoom as the main line, content selection as the auxiliary line.

The national large-scale general atlas based on the national basic database design highlights the strategic height, resource advantages and era characteristics of the atlas. The new century edition of the National Fundamental Geographic Atlas is one of 
the successful examples of comprehensive application and deep mining of national basic database resources.

At the same time, the project explores the relationship between the science cognition and aesthetic feeling on map, which makes the National Fundamental Geographic Atlas reached a high unity of science and art. In the overall decoration and beautification of the atlas, the basic colors such as Chinese red, porcelain blue, glass orange, emerald green and Great Wall grey are collected and reconstructed, so that the cultural connotation of the color elements perfectly matches the expression of the map theme, and the visual effect of the map language is improved by combining the macro and micro beautification design, which significantly improves the aesthetic value of the atlas. There are three form achievements of database, paper version and network version.

The design ideas and research results of this paper will have important reference value for other atlas to improve the innovation level of topic selection and planning. On the basis of the research results, if we continue to get the support of the national science and technology plan project, we will further deepen the research on the basic research of map knowledge, big data mining of social economy, knowledge discovery of time and space, virtual representation of atlas and other technologies, constantly enrich and update the data content, and establish a comprehensive system covering natural environment, social economy, ecological civilization and other aspects of information. With the advantages of electronic map, network publishing platform and mobile terminal, the national general atlas can be upgraded to a national large-scale comprehensive atlas, so that it can better meet the needs of China's economic construction, national defense construction, social development and ecological protection services.

\section{ACKNOWLEDGEMENTS}

This paper was suportted by the basic work special for national science and technology (2013FY112800). The authors would like to express appreciations to colleagues in our project team for their valuable comments and other helps.

\section{REFERENCES}

WANG Guizhi, WANG Donghua, ZHOU Wen, ZHU Xiuli, The Studying of the National General Atlas of China, the 28th International Cartographic Conference(ICC2017), Washington, D.C., USA, July 2-7,2017.

LU Yongsen, Editing Design Characteristic on Sequence Map of National General Atlas, Map, 1997(4):22-24.

ZHU Gorui, GUO Lizhen, YIN Gongbai, etc. Map Design and Compilation, Wuhan University Press, December 2004.

CHEN Yu, Modern Atlas Design and Research, Science Press, 2005.

DENG Guochen, Map Culture and Its Values - an interview with Academician Wang Jiayao, Surveying and Mapping Science, 2014, 39 (12): 3-7

XV Shaoshi, National New Urbanization Report, China Planning Press, 2016.
YU Liansheng, Cultural Connotation and Social Cultural Value of Maps, Bulletin of Surveying and mapping, 1998 (1): 27-29.

WANG Guizhi, WANG Donghua, ZHOU Wen, etc. The Research and Compilation of City Maps in the National Fundamental Geographic Atlas of the People's Republic of China, The International Archives of the Photogrammetry, Remote Sensing and Spatial Information Sciences, Volume XLII-3, 2018.

WANG Guizhi, WANG Donghua, QIAO Junjun, ZHOU Wen, Establishment and Compilation of Provincial Fundamental Geographic Map Database, Geomatics World, 2016(3): 87-90.

WANG Guizhi, WANG Donghua, ZHOU Wen, CHEN Mian, FENG Yu, Visual Design and Aesthetic Research of the New Century Edition of National Fundamental Geographic Atlas of China, Geomatics World, 2019(6): 64-70. 